Flows, Circulations and their Opposites: Ethnographic Perspectives and Theoretical-Methodological Challenges

Categories, people and State

\title{
Una experiencia de interiorización: transformaciones y continuidades de las acciones humanitarias
}

\author{
Angela Facundo Navia' \\ 'Departamento de Antropologia, Programa de Pós-graduação em Antropologia Social (PPGAS), \\ Universidade Federal do Rio Grande do Norte, Natal/RN, Brasil
}

\section{Resumen}

Este artículo analiza algunos aspectos del programa brasileño de Interiorización de personas procedentes de Venezuela, a partir de la experiencia de algunos "núcleos familiares" que fueron trasladados de Roraima a Rio Grande do Norte en 2018. El texto propone una comparación del programa de interiorización, en sus contornos locales en Caicó, con el programa de reasentamiento solidario previamente investigado en otras regiones del país, sugiriendo que existe una continuidad de algunas tradiciones administrativas de personas en éxodo y, al mismo tiempo, indicando transformaciones significativas en la administración de los asuntos migratorios en los últimos años. El artículo concluye con la reflexión de algunos desafíos políticos y éticos que aparecieron durante los períodos de observación participante que dieron origen a esta contribución.

Palabras clave: interiorización; reasentamiento; acciones humanitarias; Caicó; Rio Grande do Norte. 


\title{
Uma experiência de interiorização: transformações e continuidades das ações humanitárias
}

\section{Resumo}

Esse artigo analisa alguns aspectos do programa brasileiro de interiorização de pessoas procedentes da Venezuela a partir da experiência de alguns "núcleos familiares" que foram transferidos de Roraima para Rio Grande do Norte no ano de 2018. O texto propõe uma comparação do programa de interiorização -nos seus contornos locais em Caicó- com o programa de reassentamento solidário previamente pesquisado em outras regiões do país, sugerindo que existe uma continuidade de algumas tradições administrativas de pessoas em êxodo e, ao mesmo tempo, apontando transformações significativas na administração dos assuntos migratórios nos últimos anos. $\mathrm{O}$ artigo conclui com a reflexão de alguns desafios políticos e éticos que surgiram durante os períodos de observação participante que deram origem a essa contribuição.

Palavras-chave: interiorização; reassentamento; ações humanitárias; Caicó; Rio Grande do Norte.

\section{An experience of interiorización: transformations and continuities of the humanitarian actions}

\begin{abstract}
This article analyses some aspects of the Brazilian program of Interiorization of people from Venezuela based on the experience of some "family nuclei" that were transferred from Roraima to Rio Grande do Norte in 2018. The text proposes a comparison of the internalization program, in its local outlines in Caico, with the solidarity resettlement program previously researched in other regions of the country, suggesting that there is a continuity of some administrative traditions of people in exodus and, at the same time, pointing out significant transformations in the administration of migratory affairs in recent years. The article concludes with the reflection of some political and ethical challenges that arouse during the periods of participant observation that gave rise to this contribution.
\end{abstract}

Key words: interiorization; resettlement; humanitarian actions; Caicó; Rio Grande do Norte. 


\title{
Una experiencia de interiorización: transformaciones y continuidades de las acciones humanitarias
}

\author{
Angela Facundo Navia
}

\section{Introducción}

En septiembre de 2018, los periódicos locales anunciaron la llegada a Rio Grande do Norte de las primeras familias venezolanas que vendrían como refugiadas para instalarse en Caicó, una ciudad de aproximadamente 68 mil habitantes localizada en la región del Seridó. Su llegada fue entendida, coordinada y ejecutada como un proceso de "interiorización de refugiados"; uno de los ejes de la llamada Operação Acolhida. ${ }^{1}$ Dicha operación es promovida por el gobierno brasileño con el apoyo técnico y financiero del Alto Comisionado de las Naciones Unidas para los Refugiados (ACNUR); la Organización Internacional para las Migraciones (OIM); el Fondo de Población de las Naciones Unidas (UNFPA); y el Programa de las Naciones Unidas para el Desarrollo (PNUD), con el fin de "ofrecer asistencia de emergencia a los inmigrantes venezolanos que entran a Brasil por la frontera con Roraima"2. Además de esas agencias, también participan diversas ONGs que se ocupan, entre otras cosas, de la recepción y de la llamada "integración local" (Hamid, 2019) de las familias en varios estados brasileños.

En términos regionales, la interiorización significó un cambio en las tendencias de ubicación de sujetos refugiados, transferidos por programas gubernamentales que incluyen la decisión sobre sus lugares de destino. En el caso de los programas de reasentamiento, por ejemplo, regularmente fueron escogidas ciudades de las regiones Sul y Sudeste, y en menor medida, de la región Centro-Oeste. De este modo, con el actual programa de interiorización, se involucraron por primera vez comunidades locales del Nordeste, que no se habían relacionado con refugiados. Aunque no todas las personas que llegaron a Caicó eran reconocidas jurídicamente como refugiadas -pues algunas habían optado por la residencia temporal- esa categoría fue usada para referirlas en todas las noticias vehiculadas sobre su llegada: más que la idea de un simple migrante o de un extranjero (que ya era llamativa para algunos locales), fue la categoría refugiado la que pareció causar mucha curiosidad y un cierto encantamiento.

En el estado de Rio Grande do Norte ésta fue una experiencia singular, debido no tanto a la inexistencia de refugiados en el estado, cuanto al hecho de ser la única reciente con presencia colectiva y numéricamente significativa. Más de una década antes, algunos "núcleos familiares" colombianos habían llegado por medio del Programa de Reasentamiento Solidario, pero éste fue desactivado y el episodio, que incluyó sólo algunas ciudades, no dejó grandes marcas en la memoria colectiva. La decisión de transferir las familias venezolanas desde Roraima hasta Caicó -y no llevarlas, por ejemplo, a la ciudad capital- tuvo que ver, según me explicó su director local, con la presencia de la ONG Aldeias Infantis y con su infraestructura.

\footnotetext{
1 Según las informaciones de la página web oficial del gobierno brasileño, A Operação Acolhida es (la traducción me pertenece) “una gran fuerza de tarea humanitaria ejecutada y coordinada por el gobierno federal con el apoyo de agencias de la ONU y más de 10o entidades de la sociedad civil; la operación ofrece asistencia de emergencia a los inmigrantes venezolanos que entran a Brasil por la frontera con Roraima. Desde el inicio de la crisis migratoria, se estima que más de 264 mil venezolanos entraron y permanecieron en Brasil”. Además de esa descripción general, son presentados también los tres ejes que organizan la operación: “1) Ordenamiento de la frontera - documentación, vacunación y operación de control del Ejército Brasileño; 2 ) Acogida - oferta de abrigo, alimentación y atención en salud; y 3) Interiorización - desplazamiento voluntario de venezolanos de RR hacia otras unidades de la Federación, con el objetivo de su inclusión socioeconómica”. (Disponible en: https://www.gov.br/acolhida/historico/ Última consulta: 10 de febrero de 2020).

2 Tomado de la información disponible en la página web https:/|www.gov.br/acolhida/historico/. La traducción me pertenece.
} 
El director del programa también me explicó que el ACNUR habría evaluado que, con una inversión menor que la que significaría pagar el arriendo de cada familia en un inmueble alquilado, las casas de las Aldeias podrían ser remodeladas para recibir varias familias en cada una, como de hecho sucedió. Con las casas de la ONG como destino para los primeros meses de vida en la ciudad, llegaron los primeros 16 "núcleos familiares" (65 personas): parejas heterosexuales con los hijos e hijas de esa unión. Excepto por algunas pocas personas más viejas, el grupo estaba conformado por niñas y niños pequeños, y por jóvenes entre los 20 y los 40 años.

Desde octubre de 2018 hasta comienzos del 2019 realicé algunas visitas al lugar, en compañía de Saionara de Jesús Dantas, estudiante de ciencias sociales de la UFRN y quien aceptó ser mi asistente de investigación. Nos interesaba conocer más detalles del proceso de interiorización y de su puesta en marcha en la ciudad, así como establecer los contactos para que posteriormente algunas estudiantes de pregrado pudieran realizar investigaciones en el lugar. Mi interés, mucho más que por la diferencia cultural o las especificidades del grupo de personas reasentadas - que, de hecho, era bastante heterogéneo en términos de procedencia regional - estaba orientado a conocer los meandros de la administración de sujetos en éxodo, que incluyen, entre otras cosas, la toma de decisiones sobre los lugares, los actores y los mecanismos para su gobierno, así como la evaluación de las características de los grupos, que suelen ser referidos en términos de nacionalidad, y de los territorios en donde éstos serán localizados.

Con ese propósito, realicé entrevistas más formales con algunos miembros del equipo directivo de la ONG, específicamente, con las personas que fueron designadas para trabajar en el programa de interiorización. Pues, además de su reciente papel en la integración local de refugiados, la ONG continúa ejecutando sus tradicionales programas de acogida de niños y niñas cuyos padres han perdido su custodia, como también el programa de "fortalecimiento familiar y comunitario". ${ }^{3}$ Después de las primeras entrevistas con los administradores, que fueron registradas en notas durante su desarrollo y posteriormente descritas en mi diario de campo, dediqué mucho más tiempo, generalmente en compañía de Saionara, a conversar con las personas reasentadas. No grabé ninguna de las conversaciones. Aunque a todas las personas les hice las mismas preguntas (cuánto tiempo llevaban en Brasil; cómo habían sido sus migraciones previas, sus entradas y salidas del país; su relación y conexión con amigos y parientes; el tiempo de relación con sus parejas actuales; cómo había sido la experiencia de interiorización, la ruta y los interlocutores del proceso; la selección en Roraima de candidatos y la decisión de ir para Caicó) dejé que ellas hablaran libremente, de modo que cada conversación tuvo detalles y tomó caminos que no siguieron las otras; a veces anotaba algunas informaciones en una agenda durante la conversación y siempre registraba la memoria de cada jornada en un diario de campo.

Intenté también visitar y conversar con todas las familias o, al menos, ir a todas las casas. En cada casa fueron alojadas tres familias, cada una de las cuales ocupaba un cuarto y compartía los espacios comunes con las otras. El equipo de la ONG estableció normas de convivencia, que incluían la prohibición de consumo de bebidas alcohólicas o de cigarrillos; la interdicción - para los hombres - de permanecer sin camisa en los espacios comunes de las viviendas; y la distribución de las tareas de aseo de las áreas compartidas, entre otras. En la entrada de cada una de las casas fueron pegadas listas con los nombres de cada "núcleo familiar", precedidas por el número de la casa, diseñado en cartulina colorida y un buhito, también hecho en papel de manualidades, que sostenía un letrero en español en el que se leía: "Bienvenidos hermanos y amigos. Nos agrada su presencia en este lugar".

En mi primera visita sólo pude hablar con las familias de la casa más próxima a la casa-sede de la administración. Su llegada reciente todavía conservaba la sorpresa de la experiencia y del camino. La mayoría de las personas tenía algún detalle que ofrecer para complementar la versión de quien estuviera hablando. Eso, sin contar el tiempo invertido en digresiones sobre la personalidad de los niños, anécdotas u otros temas que se 
iban derivando de la charla. Al final de la conversación - y de los juegos con las niñas y los niños que estuvieron todo el tiempo interactuando con nosotros - era ya muy tarde para presentarme a otras personas. Supe, en la siguiente visita, que circuló entre ellos la información de que habría una "profesora colombiana" estudiando su situación. Supe además, por las personas con las que ya había hablado, que otras familias también querían que yo las escuchara. Hubo algunas excepciones, personas que no mostraron interés de hablar conmigo y que, cuando fui a presentarme, no manifestaron ningún entusiasmo: por el contrario, se mostraron recelosas, por lo que no insistí en la charla y tampoco mantuvimos contacto en visitas posteriores. Con el correr de los meses, algunas personas fueron desvinculadas del programa y nuevos "núcleos familiares" fueron llegando. También tuve contacto con algunas personas llegadas en ese segundo grupo.

La mayoría de las visitas o de las salidas con personas interiorizadas las realicé en compañía de Saionara. Fue gracias a ella y al hecho de ser una antigua y querida conocida de algunas funcionarias de la ONG que se facilitó enormemente no sólo mi primer contacto para pedir las autorizaciones debidas, sino también el tránsito desburocratizado por el lugar. Además de la presencia de Saionara, de los permisos que obtuve para el trabajo y de mi presentación ante las personas, que realizara el funcionario coordinador del programa, considero que hubo otros elementos que también facilitaron la relación con ellas. En trabajos previos reflexioné con más detalle cómo las marcas de nacionalidad, idioma, género y clase social fueron elementos decisivos en el establecimiento de vínculos con refugiados colombianos (Facundo Navia, 2017).

No hay tiempo aquí para detenerme en aquel análisis, pero es importante anotar que el hecho de hablar en español hizo que rápidamente estuviéramos bromeando sobre los malentendidos lingüísticos, o las diferencias regionales en la forma de nombrar las cosas; y que el tema de la interiorización -y sus detalles- pudiera ser narrado sin los límites y el cansancio de quien tiene que buscar, en un repertorio limitado de palabras, alguna que le ayude a explicar sus emociones y experiencias. Así como yo tenía curiosidad por sus tránsitos y su decisión de ir para Caicó, ellos también querían saber sobre mi historia de migración, y no ahorraban preguntas sobre de mis experiencias de movilidad, documentación, elección de lugares y opinión de las ciudades en donde había vivido en Brasil. Todo ello hacía de las charlas un momento mucho más animado y menos parecido al formato de una entrevista.

Otro elemento, que no había previsto pero que me impactó desde las primeras charlas, fue el hecho de que sus historias de migración previas se parecían mucho a las hechas por algunos parientes y amigos. Las relaciones históricas entre habitantes de Colombia y de Venezuela, y los parecidos culturales de algunas regiones de frontera, surgieron rápidamente en nuestras conversaciones. No sólo discutimos las mejores y peores opciones para hacer arepas y hallacas, y los peores, y mejores, artistas musicales del momento (en lo que hubo casi tanta divergencia como en términos de política), si no que empecé a saber sobre las temporadas que algunos de ellos, o sus familiares, pasaron en territorio colombiano. Muchas veces me entusiasmé además contando las historias de parientes que también fueron y volvieron de Colombia a Venezuela. De los sobrinos políticos venezolanos y de cómo fueron bien recibidos los que migraron pequeños, en las épocas de bonanza en Venezuela, mientras el Caribe colombiano, tal como ahora, se desangraba a manos de los paramilitares y sufría con falta de comida, médicos, escuelas y empleo.

Por otra parte, la competencia lingüística de Saionara, su conocimiento de la ciudad y de la región, marcado por su experiencia de clase, fueron también elementos importantes en el establecimiento de relaciones. Ella ayudó a activar chips telefónicos con una llamada, que las personas no habían podido hacer por limitaciones lingüísticas; sabía dónde conseguir cosas baratas en la ciudad, o daba consejos sobre dónde se podría colocar un lugar de venta de comidas populares que fuera rentable. Además, respondía las otras muchas dudas que las personas tenían sobre la ciudad: si de verdad llovía, si había períodos de fiesta, si el turismo era como les habían dicho, cuáles ciudades quedaban cerca y eran mejores para vivir, cuánto costaba un arriendo de una casa, cuál era la diferencia en precios con los barrios más baratos en Natal, etc. En esos encuentros se deshicieron 
algunas fronteras de clase y de nacionalidad, y sin la ingenuidad de creer que desaparecieron la alteridad o las relaciones de poder que constituyen el ejercicio antropológico, sentí muchas veces más proximidad con esas personas, objeto de tantas investigaciones, que con algunas de las personas administradoras y su lógica de gestión que, en cambio, parece ser menos atractiva como objeto de estudio.

A pesar de la novedad que representó la transferencia colectiva de personas y que el destino fuera la región Nordeste de Brasil, aparecieron rápidamente y de forma muy evidente algunas continuidades con las formas de administración de refugiados que había investigado en otros momentos (Facundo Navia, 2017). Mi propósito con este texto es, entonces, identificar algunas de las diferencias, así como las continuidades, entre los programas de reasentamiento de las dos primeras décadas del siglo XXI y el de interiorización de venezolanos, que comenzó en el 2018. Pretendo insistir en que los formatos político-administrativos, desplegados para vincular personas y territorios, son legatarios de tradiciones administrativas de vieja data, incluso coloniales, como señalan algunos autores (Oliveira, 2014; Souza Lima, 1995) y no responden solamente al diseño del gobierno de turno. Pero, al mismo tiempo, considero importante subrayar algunas diferencias que nos pueden alertar sobre cambios, paulatinos pero significativos, en el carácter humanitario que fue la base de las acciones para la administración de poblaciones refugiadas en el mundo desde finales del siglo pasado (Fassin, 2010).

Finalmente, destaco que las consideraciones que presento corresponden al análisis de algunos aspectos de la interiorización en sus contornos locales en Caicó. O sea, adhiriendo a la propuesta de las etnografías de lo particular (Abu-Lughod et al., 2018:206-208) pretendo reflexionar sobre una experiencia local y específica, que no agota la complejidad de experiencias del mismo programa en otras regiones, ni explora en profundidad todos los meandros administrativos o las consecuencias sociales y políticas que éste pueda traer, para las personas que en él participan o para la vida política y diplomática de Brasil, y sus relaciones con otros países de América Latina.

\section{Recepción y la esperanza de otros lugares}

Un primer contraste con el que quisiera comenzar, en parte debido a su esperanza potencial, tan esquiva en estos días, tiene que ver con la recepción que tuvieron las primeras familias que llegaron a la ciudad. No establezco dicha oposición con respecto a otros programas o formatos de administración de poblaciones en éxodo, sino respecto de la experiencia por la que pasaron, en el estado de Roraima, la mayoría de las personas del primer grupo de familias interiorizadas, antes de aceptar su traslado a Caicó. Durante los meses previos, diversas manifestaciones xenófobas marcaron la dinámica de Pacaraima y de Boa Vista, incluyendo un linchamiento, la destrucción con fuego de campamentos improvisados, golpizas y amenazas de muerte a los recién llegados. Además de los episodios violentos más evidentes, que fueron difundidos y criticados en los periódicos y noticieros nacionales, la tensión cotidiana, según las narraciones de las personas con las que hablé, iba en aumento, y la situación empeoraba con la llegada constante y masiva de más gente procedente de Venezuela. Sin embargo, después de un largo viaje, fueron sorprendidas por la recepción afectuosa preparada por algunas personas en Caicó, que se formaron a lado y lado de la vía por la que pasaría el bus y, en medio de aplausos y gritos de bienvenida, conmovieron a los viajeros y a quienes, como yo, vimos los videos por las redes sociales, compartidos por habitantes de la ciudad.

En un mundo en el que el complejo industrial fronterizo gana cada vez más fuerza y se alimenta de la xenofobia, no es un dato menor que haya ciudades en las que el impulso colectivo sea la solidaridad y el mensaje de que existe lugar para los otros. Sin la más mínima intención de hacer de ese episodio un elemento para la evaluación del éxito o fracaso del programa en Caicó, o de proponer soluciones a los graves problemas asociados a los desplazamientos en el país, sugiriendo nuevos destinos más amables, vale la pena resaltar las diferencias entre "las lógicas y sensibilidades de la acción colectiva ancladas" en lugares específicos 
(Florez, 2015:10), que se materializan en respuestas sociales diferenciadas ante los desafíos. Se trata, me parece, de la posibilidad de incluir las emociones, los deseos y los afectos colectivos como parte constituyente de las culturas políticas locales, en este caso, especialmente asociadas a las respuestas ante los desplazamientos forzados y las migraciones.

Repito, no se trata de romantizar el episodio de la recepción, y mucho menos de homogeneizar las diferencias sociales de la ciudad de acogida; o de crear la idea de una ciudadanía siempre receptiva que esconda las tensiones históricas derivadas de las complicadas relaciones socio-raciales de la región del Seridó, que han sido descritas por algunos autores (Cavignac, 2007; Goulart, 2016:50-64). Sin embargo, tampoco sería justo negarle su fuerza afectivo-política como una región potencialmente más dispuesta a la recepción y más solidaria con los migrantes pobres que las grandes ciudades capitales. De hecho, la desromantización del episodio fue realizada por algunos funcionarios que también se conmovieron con la buena recepción dada a las familias venezolanas, pero señalaron algunos de los límites de ese encantamiento inicial. Algunos de ellos se disgustaron, por ejemplo, con la cantidad de curiosos espiando por los huecos entre los barrotes de la reja que cerca el terreno que ocupa la sede de la ONG en donde fueron instalados.

La extrema curiosidad de los vecinos y su deseo de conocer a los refugiados, inquietaron a los administradores, quienes aumentaron las precauciones para no permitir que la dinámica de entrega de donaciones se convirtiera en un intercambio de objetos por la posibilidad de "ver de cerca un refugiado", como si se tratara de un "espectáculo de circo", según la expresión utilizada por uno de ellos. Con el paso de los meses, la relación con las primeras personas que llegaron se fue cotidianizando. Algunas amistades entabladas con las personas de los barrios vecinos, de las iglesias y con los compañeros de escuela o de trabajo, fueron generando otros vínculos, que perdieron los componentes de exotización inicial y también ganaron independencia de la administración de la ONG. Como era de esperarse en cualquier interacción social, algunas de esas relaciones fueron de solidaridad y concordia, y otras de conflicto.

Por otro lado, el asunto de las donaciones también permite identificar la permanencia y persistencia de algunas lógicas de administración de personas en situaciones precarias, que me gustaría señalar como elemento de continuidad, para los efectos comparativos de este artículo. La bienvenida, además de la calurosa recepción, se materializó en ingentes donaciones de ropa, juguetes, comida y otros artículos. La abundancia de cosas donadas, el estado de algunas de ellas y la decisión de que su selección y distribución quedara a cargo de los funcionarios del programa, hizo que los objetos permanecieran, por algún tiempo, amontonados en uno de los corredores donde funcionan las oficinas de la ONG, mientras el equipo lograba, clasificarlos, organizarlos y distribuirlos. La casa de la administración está localizada a la entrada del gran terreno que ocupa la ONG, y en la época estaba siempre frecuentada por personas interiorizadas, en busca de apoyo institucional para resolver algún asunto. De ese modo, la circulación constante de las personas administradas por los espacios de la administración les permitía ver la pila de donaciones que ellos mismos no tenían permiso de distribuir. No fue siquiera considerada una organización de las personas refugiadas, que se hiciera cargo, con criterios propios, de los objetos que les eran destinados, que sin duda incluiría la necesidad de mediar los conflictos.

Como en otros programas de atención a refugiados o de reasentamiento, estos episodios nos recuerdan que hay un gobierno de los sujetos que pasa por el gobierno de los objetos, y que la administración de la vida cotidiana de las personas asistidas también es una forma de control de sus comportamientos, de economía moral del gasto, de fortalecimiento del principio del merecimiento y de la idea de estar siendo cuidados y protegidos por funcionarios con experiencia en la gestión de vidas tuteladas. La aparición de una nueva ONG en el universo institucional brasileño del refugio, administrada por personas que nunca antes participaron de la gestión de refugiados, podría hacernos suponer que serían desarrolladas otras prácticas o formas específicas de administración y de relacionamiento con sujetos en éxodo. Pero, al contrario, lo que llamó la atención fue el parecido, en los formatos de acción y gestión cotidiana, con otros programas para refugiados 
previamente estudiados, así como con el establecimiento de relaciones de autoridad entre grupos familiares y la administración, que se corresponden con las dinámicas descritas por Vianna (2002) en su análisis sobre la custodia de menores.

De hecho, llama también la atención que la larga experiencia de la ONG haya sido con menores cuyos padres perdieron la custodia, es decir, con menores tutelados; y que esa capacidad administrativa se haya adaptado fácilmente a las necesidades de gestión de sujetos migrantes. Parte de la explicación para el despliegue de técnicas y tecnologías de gestión puede estar relacionada con la búsqueda de unificación de criterios técnicos para el tratamiento de refugiados que realiza el ACNUR, y para lo cual ofrece formaciones y entrenamientos a nivel global. Sin embargo, es posible resaltar también que hay rutinas de administración que corresponden a tradiciones históricas del Estado brasileño, y a su relación con actores clave en los programas asistenciales como la Iglesia católica o el ejército (Souza Lima, 1995) que no son destinadas con exclusividad a los extranjeros sino que, al contrario, pueden ser identificadas en diversos programas sociales de administración de poblaciones, especialmente poblaciones pobres (Swaan, 1992) y para las cuales el formato familiar es indispensable. El estudio de esas tradiciones administrativas puede ser una vía privilegiada para pensar sobre producción de ciudadanías diferenciadas, de fronteras de la nación construidas con criterios de raza, etnia, clase, género y sexualidad y para escapar del criterio nacional como diferenciador analítico per sé de los estudios migratorios, aunque sea fundamental considerar y tomarse en serio la forma en que esa categoría opera en la vida cotidiana.

Como en otros escenarios sociales de distribución de recursos escasos en un formato de control de la vida cotidiana, algunas manifestaciones de descontento empezaron a aparecer en los meses posteriores de la llegada de las familias a Caicó. En una de las visitas, dos familias de casas diferentes plantearon el tema de la comida. En tono irónico, haciendo bromas sobre el sabor desagradable de algunos de los productos recibidos, se quejaron de no poder escoger ellos mismos el tipo de alimentos que querían consumir. También hubo quejas sobre la poca cantidad de algunos productos que se acababan antes de que el equipo les llevara el siguiente mercado, y fricciones internas entre ellos, con acusaciones de que algunas personas estarían almacenando parte de la comida recibida, sin distribuirla con las otras familias de la casa. El descontento paulatino que las personas van sintiendo, a medida que el entusiasmo y el impacto de las primeras etapas del viaje y de la instalación pasan, o a medida que las expectativas no cumplidas se van convirtiendo en hechos concretos, también es un viejo conocido de los programas de administración de refugiados.

\section{Seducción y desencanto}

Vale la pena mencionar que, según las personas con las que hablé, el agravamiento de la situación en Roraima, no sólo por el horror de la violencia xenófoba, sino por las condiciones de penuria impuestas por la vida en los albergues, también tuvo mucho peso a la hora de tomar la decisión de aceptar la interiorización y de optar por los lugares ofrecidos, en este caso Caicó, así ésta no fuera la opción más deseada. La vida, que para muchos se volvió insostenible en la frontera, contribuyó una vez más a que la solución ofrecida tuviera un carácter de salvación, como bien lo señaló Vianna en relación con otros escenarios sociales (2002) y como lo sugerí a respecto del refugio de colombianos (Facundo Navia, 2017). La oferta de un futuro mejor en Caicó, según el relato de los interlocutores, estuvo acompañada de descripciones de la ciudad que señalaban su supuesta proximidad con el litoral; la existencia de un polo agroindustrial en la región, y un intenso movimiento turístico que ofrecería empleos a los hispanohablantes. A pesar de las potencialidades de la ciudad, esas informaciones no son del todo exactas y la fotografía que ayudaron a crear en la imaginación de las personas que las recibieron, contribuyó a su posterior desilusión. 
Al mismo tiempo que les describieron positivamente el lugar ofrecido para la interiorización, las personas también estuvieron presionadas por la idea de perder la última oportunidad de salir de Roraima. Según contaron algunas de ellas, la información de que era posible que no salieran más buses, o de que tuvieran que pasar muchos meses más en los albergues, antes de la reactivación de la interiorización en el 2019, fue frecuente. Una pareja, cuya hija tenía seis años, relató que la decisión de aceptar ir a Caicó estuvo, en gran parte, motivada por el llanto de la niña cuando notó que muchos de sus amigos ya usaban el brazalete que identificaba a los que estaban en las listas de embarque. La salud y el ánimo de la niña estaban deteriorados y cuando se percató de que los papás no habían aceptado los brazaletes, rompió en llanto, pidiendo que salieran del lugar. La situación hizo que cambiaran de opinión y, en lugar de esperar un próximo bus con destino a São Paulo o Rio de Janeiro, como lo deseaban, aceptaron venir a Rio Grande do Norte. Supe hace pocas semanas, por un mensaje de WhatsApp enviado por una amiga que permanece en la ciudad, que la niña y sus papás viajaron con ayuda financiera de familiares hacia Río de Janeiro, junto con su hermana, de pocos meses de nacida.

Al mismo tiempo que se insistió en el carácter temporal, provisional y limitado de los recursos, así como en las condiciones socioeconómicas difíciles de los lugares de destino, se ofrecieron también descripciones idealizadas de los lugares a los que serían llevados. Se trató de una exageración - o a veces, una distorsión - de aspectos juzgados como positivos, que buscaba, según la interpretación de las propias personas interiorizadas con las que hablé, estimular el deseo del viaje y, a veces, subirles el ánimo para que aceptaran embarcarse.

Esta práctica, aunque no sea la intención de las personas que la materializan, termina por fortalecer la idea, tan valiosa para el neoliberalismo, de una decisión libre e informada de sujetos racionales y autónomos. Esa supuesta libertad, alimentada por un deseo individual (Giddens, 1995; Rose, 1996) cumple también el papel de fortalecer la idea de formas de acción democráticas y respetuosas de las decisiones de movilidad de las personas, que contrastarían con las prácticas de detención o expulsión forzadas de otros países. A pesar de las presiones que sufrieron a la hora de tomar la decisión de aceptar la interiorización, todas las personas con las que hablé al respecto estaban de acuerdo con que había sido una decisión voluntaria, tal como se presenta en los documentos y páginas oficiales de la Operação Acolhida.

Hoy en día, algunas de las personas que llegaron con el primer grupo interiorizado están empleadas y permanecieron en la ciudad, a pesar de haberse terminado el tiempo de vinculación con el programa. Otras permanecen en la región, aunque salieron de la ciudad; y otras tantas salieron de la ciudad y de la región, en diversas circunstancias, la mayoría de las veces, autofinanciadas o con ayuda de parientes. A pesar de esas trayectorias diferentes, cuando aún era reciente la llegada del primer grupo, la mayoría manifestó que hubiera querido ir a ciudades más grandes, más conocidas, con mejores ofertas de empleo o con la presencia de otros parientes y amigos. Algunas ciudades también fueron mencionadas por ofrecer rutas mejores (más rápidas y más baratas) para eventuales viajes a otros países de Latinoamérica, donde también tenían seres queridos.

La comunicación permanente por redes sociales - especialmente WhatsApp y Facebook - con otros coterráneos alimentaba, a su vez, la idea de que otras ciudades o países eran más promisorios, y que los demás estaban logrando conseguir dinero y una vida con mayores comodidades, mientras que ellos no lograban hacerlo en la misma medida. En varias oportunidades me mostraron fotos de las casas adonde estaban viviendo esos parientes o amigos, y las comparaban con las que ellos habitaban. Especialmente con el hecho de que los otros pudieran vivir solos, y que a ellos les tocara compartir la vivienda con otras dos familias, con las que en, algunas ocasiones, además hubo desentendimientos y peleas. El otro elemento frecuentemente comparado fue la rapidez y relativa facilidad de sus conocidos para conseguir trabajo. En pocos meses, según lo que contaban los mensajes de WhatsApp que me mostraron, ya habían sido empleados con garantías contractuales, mientras que en la época de llegada del primer grupo reasentado a Caicó, solamente unos pocos hombres habían conseguido trabajos temporales y sin las garantías de ley. 
Desconozco si la información que recibían de sus conocidos se ajustaba a la realidad, o si estaba exagerada, o moderada por algún motivo. Tampoco tendría cómo saber si realmente alguna ciudad ofrece mejores condiciones de vida para todos los migrantes venezolanos con las características de estos "núcleos familiares". Es difícil suponer que un lugar pueda colmar todas las expectativas de todos los sujetos; cada ciudad o pueblo va quedando grande o pequeño para los sueños, y va volviéndose suficiente o insuficiente para las personas, no sólo por las condiciones del lugar, sino también por las características de las personas y de la situación específica de su curso vital. O sea, los arreglos en un mundo provisional, además de que son globalmente injustos, siempre son existencialmente contingentes, en especial para las experiencias de transformación profunda motivadas por el éxodo, que obligan a realizar una evaluación permanente del lugar que se habita y de aquellos que se podrían habitar.

En los primeros meses después de su llegada, por ejemplo, Roraima apareció en el relato de algunos de los interlocutores como un lugar mejor que Caicó. Incluso aquel espacio, de peligro y sufrimiento, ofrecía algunos beneficios importantes, como la abundante circulación diaria de personas y de dinero en efectivo que les permitía, inclusive sin un contrato formal de trabajo (cuidando carros o vendiendo café, según sus palabras), ganar en pocas horas sumas mucho mayores a las que habían logrado conseguir con semanas de trabajo en Caicó. Para varios de ellos la cercanía con la frontera también era un aspecto importante, pues ampliaba las opciones de idas y vueltas a Venezuela para llevar alimentos (Vasconcelos, 2018), dinero y otros productos; y a veces, para traer parientes, vender propiedades o rescatar pertenencias que no lograron cargar en los otros viajes. Esos movimientos se volvieron más difíciles cuando llegaron a Rio Grande do Norte y percibieron, en términos de costos y tiempo, qué tan lejos estaban.

Dos parejas, que llegaron con un segundo grupo a Caicó, acusaban a algunas de las personas del primero de no haber hecho lo suficiente para buscar empleo y de ser muy exigentes con las condiciones laborales que pretendían, rechazando empleos sin carteira assinada o con salarios menores que el de los brasileños. La acusación se dirigía especialmente a quienes, según ellos, no tenían parientes en Venezuela dependiendo de su fuerza de trabajo, sino por el contrario, estaban esperando que les mandaran dinero. Esa era la explicación que encontraron al porqué estaban conformes con la ciudad y con la escasa asistencia monetaria que recibían del programa, y no hacían "lo que fuera" para ganarse la vida. Tener a alguien en Venezuela que dependiera de las remesas o de los viajes para llevar productos, o tener redes, de familiares o amigos, en otras ciudades o países, fue un elemento central en las evaluaciones que las personas hicieron de los lugares considerados adecuados para conseguir sus objetivos de vida, y también de la posibilidad o no de exigir el cumplimiento de derechos laborales y condiciones de trabajo. Como bien lo ha mostrado Feldman-Bianco, los desplazamientos forzados y las expulsiones en la era del capitalismo corporativo están ampliando las desigualdades sociales y contribuyendo, en un ritmo acelerado, a la deshumanización de la pobreza (2015:20). Aquí, me gustaría detenerme, no obstante, en otro aspecto de esa relación con los parientes que se quedaron en Venezuela o que fueron a otros lugares.

\section{Los núcleos familiares, la frontera y la sexualidad de la nación}

Algunas autoras, que han enfocado en las particularidades de los procesos migratorios de personas con sexualidades no hegemónicas o personas trans, han rescatado, en textos recientes, la discusión sobre la tendencia de los estudios migratorios clásicos a concebir privilegiadamente al sujeto migrante como un hombre cisgénero, heterosexual y blanco (Andrade, 2019; França, 2017; Fonseca, 2020). Esos trabajos han enfatizado, asimismo, la necesidad de incluir las categorías sexualidad y género como ejes fundamentales en el análisis de las experiencias y procesos de movilidad. 
Evidentemente, la incorporación de los estudios de género en los estudios migratorios desestabilizó la idea de una migración mayoritariamente masculina, e incluyó las experiencias de mujeres, gays, personas trans, grupos familiares, niñas y niños desacompañados, etc., como realidades fundamentales de las dinámicas planetarias de circulación o diáspora, complejizando así el entendimiento de las migraciones contemporáneas. Sin embargo, el foco en las sexualidades no hegemónicas, a veces, deja de lado la pregunta por la construcción permanente y el refuerzo de la heterosexualidad como norma de la nación, tal como ha sido sugerido por Ochy Curiel (2013) y como una sexualidad igualmente producida - y reproducida - en las prácticas de Estado y de definición de la nación.

En los programas de refugio y, especialmente, en el de reasentamiento de nacionales colombianos durante las primeras décadas del siglo veintiuno y el actual programa de interiorización de nacionales venezolanos, el sujeto privilegiado de la migración no es, necesariamente, un individuo sino un "núcleo familiar" cisheterosexual, y mayoritariamente - aunque no exclusivamente -, blanco-mestizo. Evidentemente, otras facetas de la interiorización han enfocado en el sujeto trabajador, cuyos movimientos están vinculados a ofertas de empleo preestablecidas. Sin embargo, es la configuración del llamado "núcleo familiar" - y la presunción de su heterosexualidad- la que orienta la mayoría de los programas.

El formato familiar bajo el cual se organiza buena parte de la interiorización, no sobra recordarlo entonces, está lejos de ser una traducción literal de las múltiples realidades y configuraciones familiares diseñadas, a lo largo de la vida, por las personas a las que se destinan los programas. No solamente en términos de sexualidad sino de componentes, límites, relaciones intergeneracionales e inter-clase al interior de grupos extensos, pertenencias regionales y nacionales, etc. Podríamos decir que el modelo familiar de esos programas es, al contrario, un esfuerzo por domesticar la multiplicidad y plasticidad de la noción de familia, y de ajustarla a una forma administrable que, a su vez, responde a un modelo hegemónico de organización social, económica, cultural y moral. Además de la elección de familias heterosexuales, que ya es elocuente sobre la concepción de la sociedad y sus normas sociales, el hecho de que sean familias nucleares (el núcleo entendido como la pareja con su descendencia) también impone escogencias, separaciones y reformulaciones estratégicas de los vínculos con aquellos que son considerados parte de la familia y que resultan fundamentales para la continuación de los procesos vitales.

Por supuesto, la capacidad de las personas para recrear sus vínculos con aquellos que les son intrínsecos, con los cuales se constituyen mutuamente como personas (Sahlins, 2013), de agregar nuevos sujetos a los enmarañados de relaciones, o de reformularlos, escapa y trasciende a los propósitos administrativos de los programas y a los esfuerzos normativos más amplios. Como bien lo mostró Machado (2013) en su análisis sobre las relaciones de parentesco en otro contexto migratorio, éstas pueden actualizarse incluso en la distancia. $\mathrm{El}$ envío y circulación, de bienes y remesas, es uno de los elementos señalados por el autor como fundamentales en el mantenimiento de antiguas relaciones, en la creación de nuevos lazos y en las transformaciones de funciones, status y autoridades familiares, como también lo han descrito Teixeira (2008:296) y Assis (2003), quienes analizan diversas formas en las que, en contextos de tránsitos geográficos y de género, no sólo se definen nuevas familias y parientes, sino que se renegocian los lugares ocupados en las configuraciones familiares de origen.

A pesar de la creatividad de la que son capaces los sujetos, que les permite driblar las camisas de fuerza impuestas por formatos de gestión restrictivos y normativos, es imposible desconocer la influencia que los mismos ejercen en sus vidas. En algunos casos, de hecho, el momento de la interiorización también fue el periodo de separación de las familias. En las palabras de una de las interlocutoras: "a ellos les salió viaje para un lado y a nosotros para otro, entonces no pudimos seguir juntos". En otro caso, Dayana sólo logró juntar el dinero para traer a su hijo desde Venezuela, más de un año después de su llegada a Caicó y gracias a un préstamo que la empleadora de su esposo (padre de su segunda hija) aceptó realizarles para contribuir con 
ese proyecto. Para lograr que su hijo se fuera a vivir con ella, primero fue necesario salir de la sede de la ONG Aldeias, pues no se les permitía llevar más personas a las casas en las que el programa los instaló durante el tiempo de asistencia.

Así, si bien algunos vínculos con otros parientes del grupo familiar extenso pueden ser, por ley, considerados para la unificación familiar (especialmente en términos de permisos de entrada al país y de documentación), es casi imposible que sean considerados para las transferencias realizadas en el marco de la interiorización. Eso significa que los costos de los traslados de esos otros que se quedaron en Venezuela o que están en otros países (tíos, hermanas, padres, abuelas, primos, comadres, amigos, hijos de otros matrimonios, etc.) tienen que ser asumidos sin apoyo económico y/o social de los programas. Situación que contribuye a que las personas hagan "lo que sea" para poder juntar de nuevo a la familia o garantizar su bienestar a distancia.

De otro lado, reforzando los esfuerzos comparativos de este artículo, en el caso de interiorización estudiado, además de tales exigencias de restringir el número y el tipo de parientes que viajan juntos o que serán beneficiarios de la asistencia del programa, también llaman la atención las diferencias en materia de documentación y situación migratoria presentes en un mismo "núcleo familiar". Esto constituye una notable diferencia con el programa de Reasentamiento Solidario, en el que los esfuerzos se centraban en que todas las personas fueran reconocidas oficialmente como refugiadas. De hecho, no se podía proceder a la transferencia geográfica de las personas hacia Brasil sin antes garantizar su reconocimiento en el primer país de asilo. En ese sentido, las respuestas legales para la migración venezolana han sido más parecidas a las formas en que se administró la circulación de haitianos y haitianas, según ha sido señalado por Vieira (2019). Tal como lo describe la autora, negociaciones, encuadramientos, adaptaciones y ajustes fueron desplegándose a medida que las condiciones de las personas y las características de sus tránsitos cambiaban; a medida que nuevos actores aparecían en el escenario de las negociaciones; y a medida que las relaciones políticas entre los países de origen y de destino se modificaban.

En el primer grupo de "núcleos familiares" que llegó a Caicó, la mayoría de las personas tenía un protocolo de solicitud de refugio. Para entonces, en 2018, parecía que la respuesta que el gobierno había encontrado para lidiar con la presencia, en territorio brasileño, de personas oriundas de Venezuela era muy parecida con la que fue utilizada para aquellas procedentes de Haití, en los primeros años de esta década. La posibilidad de activar una solicitud de refugio fue la forma encontrada para dar opciones de documentación, ya que el protocolo de solicitud era la vía de acceso a otros documentos, como CPF (Cadastro de Pessoa Física), carteira de trabalho y carné de salud. Pero, tal como entonces, se presumía que el reconocimiento final no iba a suceder (Vieira 2019: 34-37), por lo que tampoco se iban a realizar las otras etapas del proceso, como las entrevistas con funcionarios del Comité Nacional para los Refugiados (Conare), o la deliberación del caso en el Comité.

La suposición de que las personas no serían reconocidas como refugiadas aumentó con la publicación de la Resolución Normativa № 126 del Consejo Nacional de Inmigración (CNIg), ${ }^{4}$ que preveía la regularización migratoria de venezolanos y venezolanas, otorgando un permiso de residencia temporal de hasta dos años. Dicha resolución, no obstante, exigía la renuncia expresa a la solicitud de refugio por parte de las personas que quisieran acogerse a esa nueva posibilidad de regularización. También esa opción había sido activada por algunas de las personas interiorizadas en Caicó, de modo que en un mismo "núcleo familiar" había diferentes estatus migratorios, que exigían procedimientos también diferenciados para la renovación de documentos y acceso a algunos beneficios.

El panorama, en términos de estatus migratorios, y la interpretación por parte de algunas organizaciones de la sociedad civil sobre lo que iba a suceder con los procesos de documentación de los solicitantes de refugio venezolanos, cambió radicalmente el 5 de diciembre de 2019. El gobierno decidió hacer un reconocimiento 
colectivo de la condición de refugiados de 21.432 solicitantes venezolanos; una cifra que representa el doble del número total de concesiones de refugio en Brasil desde 1997. Esa decisión fue recibida con beneplácito por las organizaciones de la sociedad civil y por algunos agentes locales de la OIM y del ACNUR, a quienes escuché celebrar la medida. Los elogios se basaban, en primer lugar, en el hecho de que el gobierno hubiera utilizado el criterio de "grave y generalizada violación de los derechos humanos" para el reconocimiento de los solicitantes. Esa fórmula, incorporada en la ley de refugio brasileña (Ley no 9.474/97) es muy valorada por los actores que integran dicho universo institucional, entre quienes circula su evaluación como una de las herramientas más avanzadas en materia de protección, siendo también una de las menos utilizadas por los gobiernos nacionales. En segundo lugar, la medida fue aplaudida por descongestionar la fila de procesos estancados, que aguardan una resolución en el Conare y, de esa forma, acortar los plazos de espera, tan onerosos para los solicitantes. ${ }^{5}$

Aliviar la incertidumbre sobre el futuro y traer beneficios en términos de documentación - y el acceso a servicios públicos que de ella se deriva - no es poca cosa. La inseguridad sobre el futuro, y el tiempo que las personas pasan esperando una respuesta definitiva, son fuentes de ansiedad, tristeza y profundización de las desigualdades sociales (Vianna \& Facundo Navia, 2015). Sin embargo, mirándola con más calma, la medida no parece ser una garantía de solución para todas las personas que supuestamente se beneficiarían con la decisión. Una serie de requisitos, que ella misma contiene, se transforma en obstáculos para el reconocimiento. La medida, por ejemplo, exige que las personas presenten documentos que certifiquen la nacionalidad, lo que no siempre es fácil en el caso de la migración venezolana contemporánea. Además de eso, no pueden beneficiarse las personas que hayan solicitado la residencia temporal (pues, como lo he mencionado, esa opción exigía la renuncia a la solicitud de refugio) y tampoco las personas con antecedentes penales o menores de 18 años.

Es aún temprano para poder evaluar los impactos concretos que la decisión traerá a la vida cotidiana de las personas interiorizadas, inclusive de aquellas que realmente puedan y quieran beneficiarse con la medida; $o$ para analizar los formatos burocráticos que están siendo puestos en marcha para atenderlos. Sin embargo, cabe preguntarse desde ahora cuáles serán los efectos, por ejemplo, sobre la movilidad de las personas reconocidas que, según la ley de refugio, no pueden salir del país sin el permiso del Conare, ni pueden solicitar la protección de otro país. No es difícil suponer que nuevos arreglos familiares, incluyendo a quien puede ir a Venezuela y volver a Brasil, o a quien puede ir a otros países y probar suerte, serán necesarios en esos "núcleos familiares" con estatus migratorios diferentes, y también en sus familias extensas.

Por otra parte, también me parece que debe tomarse en serio el llamado de atención de algunos estudiosos de las migraciones sobre las implicaciones políticas de la decisión gubernamental de aplicar la cláusula de "grave y generalizada violación de los derechos humanos" para juzgar la condición de refugio de las personas procedentes de Venezuela (Branco Pereira, 2020). Además, para los propósitos de este artículo, esa alerta nos ayuda a presentar algunas de las rupturas recientes más evidentes con respecto a la administración de los asuntos migratorios en Brasil, desde la época de la redemocratización hasta la segunda década del siglo XXI.

La celebración de esa medida por parte de ONGs y organismos internacionales puede contribuir a la idea de que el gobierno brasileño estaría alineado con los mecanismos e instrumentos más refinados en materia de protección de los derechos migratorios. En realidad, lo que hemos visto desde octubre de 2018 hasta ahora, ha sido el alineamiento con los principios más aterradores de la doctrina de la seguridad nacional y de la criminalización selectiva de grupos migrantes. Branco Pereira (2020), además de recordarnos la rápida salida del nuevo gobierno del Pacto Global de las Migraciones, también nos refresca la memoria sobre graves episodios de violación de principios legales, como el de no devolución de refugiados o el de obligatoriedad de protección de sus propios nacionales, cuya deportación de los Estados Unidos está contando con apoyo gubernamental y

\footnotetext{
5 No he analizado aún los impactos concretos de esa medida, en términos de documentación y acuerdos familiares, en el caso de los "núcleos familiares" que fueron reasentados en la ciudad y que aún permanecen en la ciudad. En julio de 2020, cuando este artículo ya se encontraba en la fase de revisión, el programa de interiorización en Caicó fue clausurado.
} 
que ha llevado al pronunciamiento crítico de diversas ONGs, asociaciones profesionales, entre las que se cuenta la Asociación Brasileña de Antropología (ABA) y otros actores vinculados a asuntos migratorios.

Sobre los desafíos de la situación política actual, que involucran la circulación de personas oriundas de Venezuela, me detendré al final del artículo. Por ahora, me gustaría volver al asunto de la situación migratoria diversa de esos "núcleos familiares": me parece que, además de los parecidos identificados con la forma de administración destinada a otros grupos migrantes en el pasado, también hay algunas diferencias que merecen, por lo menos, mencionarse. En primer lugar, el hecho de permitir la transferencia de grupos con situaciones migratorias diversas desde Roraima hacia otros estados, está anclada en una lectura orientada por el orden nacional de las cosas, utilizando la expresión de Malkki (1995). Es decir, en el caso del reasentamiento de colombianos, la obligatoriedad del reconocimiento previo como refugiados en Ecuador respondía a la necesidad de respetar y, simultáneamente, reforzar la idea de soberanía nacional. La frontera internacional solamente podría ser cruzada por las personas que serían reasentadas, después de que el primer país de asilo garantizara su condición de sujetos bajo protección y autorizara su salida; y también, después de que los documentos de entrada a Brasil estuvieran autorizados (Facundo Navia, 2017). La inviolabilidad de la frontera pasaba por una especie de construcción de homogeneidad en la condición migratoria de todos los integrantes del grupo, que permitiera reforzar, asimismo, la propia frontera del refugio, peligrosamente lindante con la migración económica.

A pesar de que la Operação Acolhida incluyó el despliegue de puestos de documentación en Pacaraima que privilegiaron la activación de una solicitud de refugio para la mayoría de las personas - el programa de interiorización no siempre exigió la homogeneidad de la condición migratoria para realizar la transferencia. En Caicó encontré, por ejemplo, algunas parejas que se formaron en Brasil y cuyos miembros habían cruzado la frontera con meses - y a veces años - de diferencia. Así, cada uno había vivido momentos también distintos en términos de respuestas administrativas; y sus documentos eran un reflejo de esos encuentros diferenciados con autoridades y agentes con capacidades de regularización migratoria, informados asimismo por sus tránsitos en territorio brasileño.

Finalmente, considero que el hecho de que las personas estén siendo trasladadas dentro de las fronteras nacionales, y no desde un país a otro, también permite ver una transformación en los ritmos de la transferencia, cuando se les compara con la velocidad con la que se realizaban los reasentamientos. Evidentemente, un viaje internacional, que requiere de la participación de funcionarios de otros gobiernos y de agencias internacionales, lleva más tiempo de preparación, pero esa explicación no agota todos los elementos que están en juego. Cuando en Ecuador se decretó la crisis humanitaria, derivada de las condiciones de vida de casi 60 mil refugiados colombianos (sin considerar a los solicitantes o sujetos en otras situaciones migratorias), la respuesta brasileña del reasentamiento solidario trasladó a su territorio aproximadamente 700 personas, ${ }^{6}$ desde el comienzo del programa, en 2003, hasta el año 2017. Con el programa de interiorización, desde 2018 hasta marzo de 2020, según datos de la página oficial, ${ }^{7}$ han sido trasladadas más de 27 mil personas hacia diferentes estados de Brasil, de un total de 264 mil regularizadas, no sólo en Roraima, sino en todo el país.

Al llamar la atención sobre tal contraste, no quiero decir que los ritmos de la interiorización sean adecuados a las necesidades de las personas que se acogen al programa. La idea de un buen ritmo cuando se está esperando algo - de que suceda lo que tiene que suceder en el tiempo oportuno - incluye, en sí misma, una evaluación de ajuste temporal que sólo puede ser realizada por quienes la experimentan. Sin embargo, la cantidad de personas

6 Datos obtenidos de la página oficial del CONARE 〈https://www.justica.gov.br/seus-direitos/refugio/refugio-em-numeros〉

7 <https:|/www.gov.br/acolhida/> 
interiorizadas en un tiempo relativamente corto tensiona las explicaciones que dieron los agentes, que hasta hace algunos años integraban el universo institucional brasileño del refugio, para justificar los ritmos más lentos y los números escasos del reasentamiento.

Los argumentos, recuperados grosso modo, eran la necesidad de no impactar las comunidades locales con la llegada masiva de personas culturalmente diferentes y con necesidades socioeconómicas supuestamente superiores a la capacidad de acogida de los municipios. También se argumentaba la necesidad de integración de los extranjeros, estimulando su interacción con brasileños, el aprendizaje del portugués, y evitando la formación de "guetos". Asimismo, los ritmos lentos eran positivados con explicaciones sobre la necesidad de saber escoger, tanto los lugares como el perfil de las personas, de modo que se filtraran los elementos de la guerra colombiana que, según la evaluación de los actores implicados, podrían traer el conflicto consigo. La imagen de una guerra contenida dentro de los límites geopolíticos del vecino país, pero con potencial de pasar la frontera a través de sujetos mal seleccionados, alimentaba la idea de que el proceso de filtrado era indispensable para la seguridad del territorio nacional brasileño (Facundo, 2019b).

Actualmente, sin embargo, ninguno de esos argumentos está siendo evocado para poner en duda la eficacia técnica del proceso de interiorización, o para cuestionar las posibilidades de integración de las personas y de respuesta de las comunidades locales a donde están siendo transferidas. De hecho, la Missão de Interiorização, como también es llamada en su página web oficial, señala esa velocidad como muestra de la capacidad técnica de las Fuerzas Armadas, que realizan la coordinación operacional de la Força-Tarefa Logística Humanitária, Operação Acolhida. En la página del Ministerio de Defensa, a su vez, se recuerda la vasta experiencia de las Fuerzas Armadas brasileñas en misiones humanitarias en el mundo (destacando la coordinación de la Misión de las Naciones Unidas para la estabilización de Haití - MINUSTAH) y se presenta la Operação Acolhida como la primera misión de naturaleza humanitaria dentro del territorio nacional. ${ }^{8}$ Es una forma de control político empleada para la intervención en países extranjeros, puesta en marcha para restaurar el control estatal de un territorio de frontera, cuyas autoridades locales, según las mismas páginas web, se vieron sobrepasadas en sus capacidades de respuesta.

El territorio defendido en este caso no es el de los pequeños municipios o ciudades, que reciben los llamados "núcleos familiares", amenazados por posibles guetos de extranjeros mal abrasileirados, para utilizar el concepto de Seyferth (2000). En esta ocasión, es el de la frontera con Roraima o de las ciudades cercanas, en donde se instaló parte de la "crisis venezolana", con la presencia masiva de extranjeros altamente precarizados, que desafiaron el carácter ilusoriamente brasileño de la región y la capacidad de respuesta, y control, del Estado sobre sus territorios.

Esas discrepancias en el tratamiento dado a las poblaciones extranjeras, en diferentes momentos y por diferentes configuraciones gubernamentales y estatales, tienen, sin duda, diversas causas. Sin embargo, quisiera señalar que, además de que actualmente no hay interés gubernamental en disimular el uso político que implica la figura del refugio (Branco Pereira, 2020), la arbitrariedad en la decisión de los formatos, las velocidades, los lugares y la cantidad de las transferencias en la interiorización puede estar relacionada con el hecho de que se trata de poblaciones con las cuales el Estado históricamente ha establecido relaciones de tutela. Como nos lo recuerda João Pacheco de Oliveira, a propósito de las características de esas relaciones: "Las normas nunca serán suficientes para definir una forma de acción prescrita, ya que siempre se preserva la libertad del agente para decidir de acuerdo con las circunstancias específicas y los interlocutores que en ese momento se privilegien"9 (Oliveira, 2014:144).

\footnotetext{
8 <https://www.defesa.gov.br/noticias/57698-operacao-acolhida-o-trabalho-de-militares-brasileiros-na-primeira-missao-humanitaria-em-territorionacional>

9 En el original: "No exercício da tutela as normas jamais serão suficientes para definir uma forma prescrita de ação, de vez que está sempre preservada a liberdade do agente para decidir de acordo com a especificidade das conjunturas e dos interlocutores que naquele momento vier a privilegiar" (Oliveira, 2014:144. La traducción me pertenece).
} 


\section{Ejército e iglesias como agentes de integración y algunas reflexiones político-metodológicas}

La primera visita que realicé a Caicó con Saionara coincidió con el periodo preelectoral en Brasil. Registré en mi diario de campo algunos detalles de la primera entrevista que tuvimos con los directivos locales de la ONG Aldeias, en la que yo pregunté si en las reuniones de planeación realizadas con el ACNUR, antes de poner en marcha el programa en la ciudad, habían considerado un posible escenario político de cierre de fronteras que pusiera en riesgo su continuidad. La respuesta negativa del director se basó en su confianza de que la Constitución Federal funcionaría como una talanquera contra los abusos que cualquier gobernante quisiera cometer. Con ellos, la conversación al respecto, llena de precauciones mutuas en relación a los términos y los nombres que se emplearon, se cerró sin más consideraciones. Con las personas que acababan de llegar de Roraima, al contrario, el tema aparecía con mucha frecuencia, con nombres propios y posturas enfáticas.

No recuerdo ni una sola vez - en las que el tema apareció - que las personas no se explayaran en críticas contra el gobierno venezolano, que terminaban extendiéndose en críticas contra el socialismo. Durante mis visitas a la ONG, o en las ocasiones en que salimos juntos de la sede, los jóvenes interiorizados me mostraban memes que ridiculizaban al presidente venezolano, imágenes de conciertos promovidos por cantantes colombianos en la frontera colombo-venezolana y muchísimos mensajes que circulaban por WhatsApp con noticias falsas sobre nuevas leyes o medidas implementadas, eventos políticos, manifestaciones y comportamientos moralmente reprochables de gobernantes venezolanos, que ellos defendían como verdades.

Al mismo tiempo, nos dejaban en claro, a Saionara y a mí, su predilección por el candidato del PSL (Partido Social Liberal) que, según ellos, debía ganar las elecciones para que su seguridad y la de sus familias estuviera garantizada en Brasil. Los contra-argumentos que Saionara y yo intentábamos poner en discusión fueron desestimados, sin agresividad, pero nunca sin emotividad. Todo el tiempo, a pesar de la amabilidad de las personas, tuve la sensación de que el par que conformábamos, con marcas muy particulares de estéticas, acentos, nacionalidades, raza, edad y profesiones, no hacía de nosotras interlocutoras con legitimidad para proponer lecturas políticas sobre el país. Al final, ¿cuál es el área de competencia de una profesora colombiana, blanca y capitalina, que no conocía Caicó (con una profesión que nunca es completamente entendida, como la antropología) y que llega al lugar acompañada de una joven estudiante negra, que se comunica en portugués y que no se alinea con los modelos de comportamiento de otras profesiones que suelen hacer parte de los equipos de atención psicosocial, como abogadas, psicólogas o médicas? De alguna manera, la proximidad que construimos, en algunos aspectos, era también un motivo para considerar nuestras lecturas como una opinión política cualquiera, sin más veracidad o legitimidad que la suya.

Uno de los argumentos más frecuentes para refutar nuestras lecturas políticas fue el hecho de que los militares les hubieran, en sus palabras: "explicado la realidad del país"; y que esa explicación contradijera nuestros argumentos. Además del contacto con los soldados encargados de la seguridad y otras tareas cotidianas en los albergues, el primer grupo interiorizado tuvo tiempo, durante el largo viaje desde Roraima hasta Pernambuco en avión, y después en bus, hasta Caicó, de escuchar las explicaciones sobre el devastador futuro que le esperaba a Brasil en caso de que el candidato del PT (Partido de los Trabajadores) - que fue presentado como comunista - ganara las elecciones. La amenaza era muy clara y fácil de entender para las personas que la escucharon y que nos la trasmitieron a Saionara y a mí: si él gana, Brasil se transformará en una Venezuela.

Para las personas con las que hablamos sobre la situación política de ambos países, los militares, al contrario de nosotras, eran figuras legítimas para leer la dinámica política y para prever los posibles futuros de la nación. Su profesión, completamente inteligible para los refugiados, su poder de acción materializado en aviones, alimentación, armamento y campamentos, y la autoridad moral de su lectura del mundo - como defensores de los mismos ideales compartidos de familia y de valores sociales - eran imbatibles, contra nuestros argumentos a favor de pensar otras formas de organizar la vida familiar y política, y nuestra inconstante, y precaria presencia. Espero que la gran cantidad de investigadores sociales en Roraima nos traiga, muy pronto, 
informaciones etnográficas sobre lo que significa la presencia del ejército en la vida cotidiana en los albergues y las ciudades que son objeto de esa misión humanitaria. Me parece que su papel puede ser considerado, entre otras cosas, como el de nuevos agentes de integración de refugiados, con la fuerza simbólica de representar a un Estado que los recibe en su territorio, con el despliegue material y logístico capaz de coordinar su proceso de recepción, abrigo e interiorización. Esos agentes les ofrecen, además, las cartas de navegación para interpretar las nuevas realidades locales a las que se exponen.

Si refutar los argumentos reforzados por militares resultaba complicado, más difícil resultó buscar una forma de cuestionar algunos de los argumentos de las iglesias que comenzaron a frecuentar a las familias; a veces como continuación de sus vínculos previos con ellas, en otros lugares; y a veces como vínculos nuevos derivados de esas visitas que los evangelizadores realizaban a la sede de la ONG. Si discutir los argumentos de los militares era un asunto de "opinión política", siempre susceptible de interpretaciones, el debate de los argumentos religiosos no tenía cabida. No se discute la creencia de los otros; no sólo las personas zanjaban la discusión con un argumento de fe, sino que yo misma me sentía éticamente impedida de contradecir, con el mismo énfasis que lo haría con un argumento inscrito en el dominio de la política.

Actualmente, hay algunos proyectos de investigación, de educación y de servicios, vinculados a la Universidad Federal de Rio Grande do Norte, que buscan apoyar los procesos de recepción e integración de los refugiados. Incluso fue creado recientemente el Comité Estadual Intersectorial de Atención a Refugiados, Apátridas y Migrantes (CERAM-RN) que rápidamente se ha convertido en una referencia para el debate sobre el asunto en término de políticas públicas en Rio Grande do Norte -y del cual hace parte Cáritas Arquidiocesana-. No obstante, en la época de la llegada de los primeros grupos de reasentados venezolanos a Caicó, en medio de la novedad, no hubo una respuesta -laica, popular o gubernamental - rápida que permitiera disputar los contornos y los contenidos de la ciudadanía de esos recién llegados, o de incluirlos en debates sociales amplios y proyectos de formación, social o política, no vinculados a proyectos religiosos, que posibilitaran repensar los objetivos de la integración social, tan anhelada por los programas de refugio.

Por supuesto, no estoy diciendo que las iglesias no puedan participar de esos proyectos, ni desconociendo que su trabajo de base es, muchas veces, más organizado y persistente que el de organizaciones sociales o proyectos institucionales de los gobiernos locales. De hecho, los programas de refugio, como fue discutido en otros momentos (Facundo Navia, 2019a) son históricamente implementados en Brasil por ONGs vinculadas a la Iglesia Católica. Por otra parte, gracias al vínculo con iglesias, generalmente neo-pentecostales, algunas personas reasentadas en Caicó consiguieron empleos y apoyos materiales. Estoy queriendo solamente llamar la atención de que algunas iglesias, a través de sus evangelizadores y de sus cultos, reafirmaban también el carácter de normalidad y ejemplaridad de la heterosexualidad; defendían la necesidad de que los refugiados se vincularan en una cruzada contra los intentos homosexualizadores de la izquierda continental y se alinearan con un proyecto político defendido por el entonces candidato del PSL. Así, para mí la preocupación sobre la suerte de las poblaciones refugiadas en Caicó se inscribió también en el debate, ya realizado por varios autores, sobre el carácter de las comunidades políticas que, al mismo tiempo, están en una situación de dominación y se expresan como una fuerza política potencialmente reproductora de esa misma situación (Butler \& Spivak, 2007).

Cada vez que dejaba la ciudad, durante las cuatro horas del camino de vuelta hasta Natal, me asaltaba la misma duda que me había atormentado en otras investigaciones. ¿Será que es éticamente necesario discutir los asuntos políticos con las personas con las que nos involucramos en nuestras investigaciones? ¿Sería esa una garantía de que ellas tomen la decisión libre e informada de querer o no contribuir con la investigación? ¿O no sería esa otra de las trampas de nuestra fe, liberal y moderna, en el individuo libre y consciente? Durante el trabajo de campo de otras investigaciones, ya había tomado la decisión de no siempre discutir los pormenores de la política nacional colombiana e, inclusive, de quedarme callada ante opiniones que consideraba racistas o misóginas, por ejemplo. Aun con la ganancia de no tensionar las relaciones con personas que me estaban 
ayudando a realizar mi investigación - y por algunas de las cuales sentía afecto - nunca dejé de preguntarme si esa renuncia al debate político no era una especie de engaño; o si, al contrario, era una preocupación sólo de mi parte, que obedece a la importancia que le otorgo al asunto, mientras que para otras personas hay jerarquías diferentes de los elementos que deben existir, en las relaciones sociales en general y en la relación con una investigadora social específicamente.

La única especie de certidumbre etnográfica que puedo registrar aquí es que, silenciar los argumentos de la política internacional en las conversaciones con las personas, no sólo a veces es un alivio, sino que abre la posibilidad de crear otro tipo de comunicación y entendimiento de la realidad. Recuerdo que en los momentos en que aparecía el tema de los gobiernos de Venezuela y Brasil, el discurso del hambre también surgía en su formato de narrativa congelada (Das, 1991); es decir, no para describir la desgracia concreta que es no tener qué comer, sino como argumento generalizante, universalizado y vacío, que resumía la situación de Venezuela y que se erguía como límite moral irrefutable -y como muestra de la maldad del gobierno-. Al contrario, hay experiencias que no se inscriben en el mismo registro narrativo y que pueden ser mejores vías, para entender las posiciones políticas y las decisiones vitales de las personas, entre las que se encuentra la decisión de migrar.

Un día, en la puerta de un hospital de Caicó, sentada con Dayana y su hijita, esperando nuestro turno para entrar a conocer la bebé recién nacida de Gladys, empezamos a hablar de los nacimientos, como lo ameritaba el momento. Cada una recordó las narraciones que nuestras mamás hacían de nuestro propio nacimiento, y también el de primas y hermanos, hasta que ella llegó al de sus hijos. El primero de ellos, hace 14 años, es una historia de tranquilidad, buena atención y cuidados. El de su hija de dos años, en cambio, es el recuerdo reciente de humillación, hambre y maltrato. Su hija tuvo que permanecer en el hospital y ella, en cambio, fue obligada a firmar el alta, pues no disponían de camas. Sin dinero para ir y volver del barrio donde vivía hasta el hospital, tuvo que instalarse por varios días en la puerta del hospital, durmiendo en cartones, para poder amamantar a su hija mientras estaba internada. No pude dejar de pensar que el primer nacimiento coincidió con el periodo de bonaza económica y despliegue de la política social en Venezuela; mientras el segundo coincide con el desplome de la legitimidad del proyecto chavista, con la crisis económica del país y con el bloqueo impuesto por el gobierno de los Estados Unidos, secundado por otros países. Sin embargo, en ese momento emocionalmente tan duro, esa información no tenía sentido para Dayana y, con seguridad, hubiera estorbado y ofendido ese instante de memoria. Entendí que el actual gobierno, para ella, va a llevar siempre la marca de esos días en la calle y del difícil proceso de sobrevivir que se desencadenó desde entonces.

Tratar de entender el dolor subjetivamente vivido y la forma en que las experiencias crean subjetividades, y vínculos políticos, no significa, sin embargo, que la experiencia individual pueda elevarse al estatus de argumento de fe, que no admite discusión. Si bien es cierto que hay desabastecimiento y miseria en Venezuela, también es cierto que la responsabilidad no es solamente de su gobierno; así como también es cierto que el hambre no es sólo descripción sino también producción. Creo que debemos preguntarnos no solamente por lo que el hambre produjo en la vida de las personas, sino también por los múltiples efectos que están siendo producidos con ella en los países de la región.

A pesar de esa única convicción, creo que las investigaciones con personas precarizadas, que no se encuadran en nuestros esquemas de valores, anticapitalistas y antipatriarcales, nos dejan más preguntas que respuestas ¿Cómo entender que la desinformación que nosotras, como investigadoras, percibimos, es uno de los efectos de la desigualdad y la dominación, sin que eso nos lleve a colocar a las personas en el lugar de incapaces e idiotas útiles que se dejan engañar? ¿Y cómo entender cuándo nosotros también estamos haciendo el papel de idiotas útiles, en nombre del respeto a la diversidad de ideas que tanto defendemos? ¿Qué hacer cuando las personas nos convencen de que sus valores y su fe contradicen nuestros principios éticos, como antropólogos, en defensa de la diversidad y búsqueda de equidad social? 
Cada situación etnográfica encontrará respuestas diferenciadas a los desafíos concretos que vayan apareciendo. Pero aunque no sean directamente con las personas con las que entablamos nuestras discusiones políticas en campo, éstas aparecerán inevitablemente en nuestros textos, encuentros académicos y otros tipos de eventos (muchas veces organizados para tratar de sensibilizar a las autoridades y comunidades locales sobre las necesidades de esas poblaciones). Ahí, inevitablemente, tendremos que asumir la responsabilidad política de nuestra producción y el riesgo de fortalecer lecturas del mundo que fomenten, en nuestro caso de estudio, la militarización de la vida política en la región. Evidentemente, no tengo respuestas a las preguntas que estoy escribiendo, y que muchas otras y otros colegas ya se hicieron; sólo quisiera reforzar la necesidad de hacer pública esa reflexión, de discutir estos asuntos con las y los estudiantes que, en parte por culpa nuestra, tienen una visión romantizada de las poblaciones subordinadas y de la propia práctica antropológica, en sus encuentros cotidianos con quienes sufren los rigores del capitalismo, cada vez más asustados con el socialismo.

\section{Consideraciones finales}

Este artículo buscó identificar algunas transformaciones y continuidades de las acciones humanitarias de atención y recepción de refugiados en Brasil, a través de la comparación entre el programa de Reasentamiento Solidario de colombianos y una experiencia contemporánea, en Caicó, del Programa de Interiorización de venezolanos. Para ello, se discutieron los parecidos y continuidades en los formatos de administración, marcados por el establecimiento de relaciones de autoridad y tutela, la persistencia en el modelo familiar nuclear y heterosexual como sujeto privilegiado de los programas y sus desdoblamientos en el refuerzo de un ideal de nación.

En términos de diferencias y transformaciones, fue destacado el hecho de involucrar, por primera vez en la historia reciente del país, comunidades del interior de los estados de la región Nordeste del Brasil como comunidades receptoras de refugiados, discutiendo las potencialidades de Caicó como lugar de destino. Como ruptura drástica, en términos de orientación política de los llamados programas humanitarios, fueron mencionados: la salida reciente de Brasil del Pacto Global de las Migraciones; los episodios de violación de principios legales, como el de no devolución de refugiados; y la colaboración del gobierno en procesos de expulsión de brasileños indocumentados de los Estados Unidos. Asimismo, como punto de contraste, fueron señaladas las diferencias en las velocidades de transferencia de personas cuando la crisis que se pretende remediar está dentro del territorio nacional (Roraima), y no del otro lado de la frontera, o en otros países, como sucedió en el caso de los colombianos.

Finalmente, fue discutido el papel de integración de las Fuerzas Armadas, y de las relaciones de algunas de las personas interiorizadas con las iglesias pentecostales en los procesos de integración, que incluyen modelos de ciudadanía, posicionamientos políticos y visiones de mundo. Al mismo tiempo, fue propuesta una reflexión de cómo la situación descrita impone desafíos ético-metodológicos para la antropología, en su relación con poblaciones precarizadas que, al mismo tiempo, defienden formatos conservadores y jerarquizados de sociedad.

Sumisión: 04/03/2020

Aprobación: 29/07/2020

Revisión: Federico Lavezzo 


\section{Referencias bibliográficas}

ABU-LUGHOD, Lila; REGO, F. C. V. S. do \& DURAZZO, L. 2018. "A Escrita contra a cultura". Equatorial Revista do Programa de Pós-Graduação em Antropologia Social, 5(8): 193-226.

ANDRADE, Vítor Lopes. 2019. Refúgio por motivos de orientação sexual. Um estudo antropológico na cidade de São Paulo. Florianópolis: Editora da UFSC.

ASSIS, Gláucia de Oliveira. 2003. "De Criciúma para o mundo: gênero, família e migração". Campos - Revista de Antropologia, [S.1.]3: 31-50.

BRANCO PEREIRA, Alexander. 2020. "Os usos e abusos políticos do refúgio". [online] Nexo. Disponible en https:/|www.nexojornal.com.br/ensaio/2020/Os-usos-e-abusos-pol\%C3\%ADticos-do-ref\%C3\%BAgio Acesso em 01/03/2020

BUTLER, Judith; SPIVAK, Gayatri. 2007. Who Sings the Nation-State? Language, Politics, Belonging. Oxford: Seagull Books.

CAVIGNAC, Julie. 2007. Relatório Antropológico da comunidade quilombola de Boa Vista (RN). Natal: UFRN/ INCRA-RN.

CURIEL, Ochy. 2013. La nación heterosexual. Análisis del discurso jurídico y el régimen heterosexual desde la antropología de la dominación. Bogotá: Brecha Lésbica/ En la frontera.

DAS, Veena. 1991. "Composition of the personal voice: violence and migration". Studies in History, 7(1): 65-77.

FACUNDO NAVIA, Angela. 2017. Êxodos, Refúgios e Exílios: Colombianos no Sul e Sudeste do Brasil. Rio de Janeiro: Papéis Selvagens.

FACUNDO NAVIA, Angela. 2019a. "Encontros produtivos: fazendo estado e refugiados". In: Carla Costa Teixeira; Andréa Lobo \& Luiz Eduardo Abreu (orgs.), Etnografia das instituições, práticas de poder e dinâmicas estatais. 1a ed. Brasília: ABA. pp. 25-52.

FACUNDO NAVIA, Angela. 2019b. "Deslocamento forçado na Colômbia e Políticas de refúgio no Brasil: Gestão de populações em êxodo". Revista de Estudos e Pesquisas Sobre as Américas, 13: 245-267.

FASSIN, Didier. 2010. La Raison Humanitaire: une histoire morale du temps présent. «Hautes études». Paris: Éditions de l'EHESS (avec Le Seuil/ Gallimard).

FELDMAN-BIANCO, Bela. 2015. "Apresentação: deslocamentos, desigualdades e violência do estado". Ciência e Cultura, 67(2): 20-24.

FLOREZ, Juliana. 2015. Lecturas emergentes. El giro decolonial en los movimientos sociales. 2aㅡ ed. Bogotá: Editorial Pontificia Universidad Javeriana.

FONSECA, Nathália. 2020. Entrecruzamentos entre migração, gênero e sexualidade: experiências de vida de mulheres não-cisheterossexuais venezuelanas e solicitantes de refúgio. Disertación de Maestría, defendida en el Programa de Posgrado en Antropología de la Universidad Federal Fluminense (UFF), Brasil.

FRANÇA, Isadora Lins. 2017. "Refugiados LGBTI: direitos e narrativas entrecruzando gênero, sexualidade e violência”. Cadernos Pagu, 50: e17506.

GIDDENS, Anthony. 1995. "La trayectoria del yo". En: Modernidad e identidad del yo: el yo y la sociedad en la época contemporánea. Barcelona: Península. pp. 93-139.

GOULART, Bruno. 2016. Nego veio é um sofrer: representação e subalternidade numa irmandade negra do Seridó. Natal: EDUFRN.

HAMID, Sônia. 2019. (Des) Integrando Refugiados: os processos do reassentamento de palestinos no Brasil. Brasília: Editora UnB

MACHADO, Igor. J. R. 2013. "Parentesco e diferencialidades: alternativas à identidade e às fronteiras étnicas no estudo das migrações”. In: Bela Feldman-Bianco (org.), Desafios da antropologia brasileira. v. 1. 1a ed. Brasília: ABA. pp. 153-173. 
MALKKI, Liisa. 1995. Purity and Exile: Violence, Memory, and National Cosmology among Hutu Refugees in Tanzania. Chicago: University of Chicago Press.

OLIVEIRA, João Pacheco de. 2014. “Pacificação e tutela militar na gestão de populações e territórios”. Mana. Estudos de Antropologia Social, 20(1): 125-161.

ROSE, Nikolas. 1996. “Governing 'advanced' liberal democracies”. In: Andrew Barry; Thomas Osborne \& Nikolas Rose (eds.), Foucault and political reason: liberalism, neo-liberalism and rationalities of government. Chicago: The University of Chicago Press. pp. 37-64.

SAHLINS, Marshall. 2013. What kinship is... and is not. Chicago: The University of Chicago Press.

SEYFERTH, Giralda. 2000. "Assimilação dos Imigrantes no Brasil: Inconstâncias de um Conceito Problemático". Revista Travessia, 36: 44-50.

SOUZA LIMA, Antonio Carlos. 1995. Um Grande Cerco de Paz. Poder tutelar, indianidade e formação do Estado no Brasil. Petrópolis: Vozes.

SWAAN, Abram de. 1992. A cargo del Estado. Barcelona: Pomares-Corredor.

TEIXEIRA, Flávia do Bonsucesso. 2008. "L'Italia dei Divieti: entre o sonho de ser europeia e o babado da prostituição". Cadernos Pagu, 31: 275-308.

VASCONCELOS, Iana dos Santos. 2018. "Receber, enviar e compartilhar comida: aspectos da migração venezuelana em Boa Vista, Brasil”. REMHU, Rev. Interdisciplinar da Mobilidade Humana, 26(53): 135-151.

VIANNA, Adriana \& FACUNDO NAVIA, Angela. 2015. "Tempos e deslocamentos na busca por justiça entre 'moradores de favelas' e 'refugiados"'. Ciência e Cultura, 67(2): 46-50.

VIANNA, Adriana. 2002. Limites da menoridade: tutela, familia e autoridade em julgamento. Tesis doctoral, Universidad Federal de Río de Janeiro, Museo Nacional, Programa de Posgrado en Antropología Social (PPGAS/MN/UFRJ), Brasil.

VIEIRA, Rosa. 2019. "Mudando peças: o jogo de enquadrar pessoas em circulação". Em: Facundo et al. (orgs.), Pessoas em movimento. Práticas de gestão, categorias de direito e agências. Rio de Janeiro: Fundação Casa de Rui Barbosa / 7 Letras. pp. 29-49.

\author{
Angela Facundo Navia \\ Department of Anthropology, Graduate Program in Social Anthropology - PPGAS, Federal University \\ of Rio Grande do Norte, Natal, Brazil \\ https://orcid.org/oooo-0001-9552-5763 \\ Email: angelafacundo@hotmail.com
}

